\title{
Usual-Type Endocervical Adenocarcinoma with a Microcystic, Elongated, and Fragmented Pattern of Stromal Invasion: A Case Report with Emphasis on Ki-67 Immunostaining and Targeted Sequencing Results
}

\author{
Sangjoon Choi ${ }^{a}$ Soohyun Hwang ${ }^{a}$ Sung-Im Do ${ }^{b}$ Hyun-Soo Kim ${ }^{a}$ \\ aDepartment of Pathology and Translational Genomics, Samsung Medical Center, \\ Sungkyunkwan University School of Medicine, Seoul, Republic of Korea; ${ }^{b}$ Department of \\ Pathology, Kangbuk Samsung Hospital, Sungkyunkwan University School of Medicine, \\ Seoul, Republic of Korea
}

\section{Keywords}

Cervix - Endocervical adenocarcinoma - Microcystic, elongated, and fragmented (MELF) pattern $\cdot$ Immunohistochemistry $\cdot$ Targeted sequencing

\begin{abstract}
The microcystic, elongated, and fragmented (MELF) pattern is a unique myometrial invasion pattern occasionally found at the invasive front of endometrial endometrioid carcinoma (EEC). Herein, we report an uncommon case of usual-type endocervical adenocarcinoma (UEA) with a MELF pattern. We comprehensively analyzed its clinicopathological and molecular features, which has not been previously documented. A 67-year-old woman presented with a cervical mass and underwent radical hysterectomy. Histologically, the MELF pattern of UEA was almost identical to that of EEC. Tumor glands exhibited a microcystic appearance or elongated structures with compression forming a slit-like lumen. The tumor glands were irregularly fragmented into small clusters or single cells. Cells lining the tumor glands possessed conspicuous eosinophilic cytoplasm with squamoid or flattened endothelium-like appearance. These glands or cells were accompanied by a prominent fibromyxoid stromal reaction. Lymphovascular invasion was occasionally observed. Immunostaining revealed diffuse and strong cytokeratin 7 expression and block p16 positivity in both conventional and MELF components. However, the MELF component displayed a very low Ki-67 proliferation index compared to that of the conventional component, which showed markedly increased Ki-67 expression. Targeted sequencing analysis revealed that the MELF component harbored pathogenic muta-
\end{abstract}


tions in ARID1A, KRAS, and PTEN, identical to those detected in the conventional component. In summary, the morphological features of the MELF pattern in UEA were similar to those in EEC. We found significant differences in Ki-67 expression status between conventional and MELF components, but the mutational profiles were identical. Our findings should be confirmed in larger cohorts of patients with UEA showing a MELF pattern.

(C) 2020 The Author(s).

Published by S. Karger AG, Basel

\section{Introduction}

The microcystic, elongated, and fragmented (MELF) pattern represents a very distinctive morphological pattern of myometrial invasion observed in endometrial endometrioid carcinoma (EEC). This pattern of invasion is typically seen at the deepest invasive front $[1,2]$. The MELF pattern has been shown to be associated with lymphovascular invasion (LVI), lymph node metastasis (LNM), and deep myometrial invasion [3, 4]. Presence of the MELF pattern in endocervical adenocarcinoma is an extremely rare condition. Currently, there has been only one study reporting on the clinicopathological and prognostic significance of the MELF pattern in the uterine cervix [2]. Furthermore, there has been no study evaluating the molecular features of endocervical adenocarcinoma that demonstrates a MELF pattern.

In this study, we present a rare case of usual-type endocervical adenocarcinoma (UEA) showing a diffuse MELF pattern in a 67-year-old woman and describe the associated pathological and molecular features. We anticipate that this report will extend the current knowledge in relation to the MELF pattern in endocervical adenocarcinoma by describing its clinicopathological characteristics, immunophenotype, and molecular characteristics obtained from targeted sequencing analysis.

\section{Case Presentation}

A 67-year-old Korean woman with no previous history of gynecological disease was referred to our institution due to abnormal cervicovaginal cytology results. A cervical punch biopsy had been performed at a local clinic and the biopsied specimen was interpreted as UEA. Abdominopelvic magnetic resonance imaging revealed a relatively well-circumscribed mass involving the endocervix (Fig. 1A). The mass measured $3.8 \mathrm{~cm}$ in diameter and showed a mixed solid and cystic appearance. No parametrial or vaginal extension was noted. Small benign-appearing pelvic lymph nodes were identified. There was no visible metastasis in the abdominopelvic cavity. Based on a clinical impression of cervical cancer, she underwent radical hysterectomy with bilateral salpingo-oophorectomy and bilateral pelvic lymph node dissection. After histological examination, she was diagnosed as having International Federation of Gynecology and Obstetrics (FIGO) stage IB2 UEA. Due to the presence of LVI and deep one-third stromal invasion (intermediate risk), she underwent postoperative radiation therapy. Three months after treatment, there was no evidence of recurrent disease or distant metastasis.

Grossly, a polypoid tumor involved the endocervix and low uterine segment. The cut surface was white-to-tan in color and solid (Fig. 1B). The tumor infiltrated into the cervical stroma, but did not involve the parametrium or vagina. The endomyometrium was unremarkable and the uterine serosa was smooth and glistening. The bilateral ovaries and salpinges were also unremarkable. Histologically, the UEA tissue exhibited a complex glandular architecture, mucin-poor glands, cribriform structures, and dilated glandular lumina

\section{Karger'}


possessing necrotic debris. The tumor cells exhibited a characteristic pseudostratified columnar epithelium with elongated and hyperchromatic nuclei. The tumor nuclei possessed conspicuous nucleoli. Mitotic figures were frequently identified, especially in the apical zone of amphophilic-to-eosinophilic cytoplasm. Apoptotic bodies and karyorrhectic debris were readily visible within the epithelium and glandular spaces (Fig. 1C). Although most areas exhibited a relative depletion of mucin and pseudostratified nuclei, mucin pools and a single layer of the columnar epithelium with appreciable intracytoplasmic mucin were occasionally noted. A MELF pattern was noted at the invasive tumor front, in which the tumor glands displayed a microcystic and elongated appearance and were lined by flattened epitheliumresembling endothelial cells. Clusters or individual tumor cells were occasionally detached from the tumor glands. Tumor cells and glands were not apparent at low-power magnification due to being obscured by striking stromal desmoplasia and the myxoinflammatory response (Fig. 1D, E). Transition from conventional UEA to the MELF component was frequently identified (Fig. 1F, G). Inflammatory cells often infiltrated around the glands and detached cells (Fig. 1H) and were observed within gland lumina. The tumor cells exhibited eosinophilic or vacuolated cytoplasm and were surrounded by fibromyxoid or edematous stroma that was associated with inflammatory cells. Tumor cells forming glands and clusters in some foci resembled histiocytes and exhibited a squamoid morphology or epithelioid morphology (Fig. 1I). Lymphovascular invasion was occasionally detected (Fig. 1J, K).

The conventional UEA component showed diffuse and strong nuclear and cytoplasmic p16 immunoreactivity (block p16 positivity; Fig. 2A), uniform cytokeratin (CK) 7 expression (Fig. 2B), and a marked increase in Ki-67 positivity (proliferation index $>90 \%$; Fig. 2C). At the invasive front, tumor glands, fragmented tumor cell clusters, and randomly scattered individual tumor cells of the MELF component were highlighted by p16 (Fig. 2D, E) and CK 7 (Fig. 2F, G) positivity. In contrast to the conventional UEA component, the MELF component displayed a very low Ki-67 proliferative activity $(<1 \%$; Fig. $2 \mathrm{H}, \mathrm{I})$.

Because of the differences in Ki-67 expression patterns between the conventional UEA and MELF pattern components, we examined the immunoreactivity in areas of transition (Fig. 3A-C) and/or LVI (Fig. 3D). In all transitional areas examined, a difference in Ki-67

Fig. 1. Imaging, gross, and histological features of usual-type endocervical adenocarcinoma (UEA) showing a microcystic, elongated, and fragmented (MELF) pattern of invasion. A Magnetic resonance imaging scan revealing a relatively well-circumscribed mixed solid and cystic mass in the endocervix showing heterogeneous enhancement. B Grossly, the endocervical tumor showed a white-to-tan solid cut surface protruding into the endocervical canal and infiltrating into the cervical stroma. $\mathbf{C}$ The UEA displayed a complex glandular architecture consisting of a pseudostratified columnar epithelium with elongated hyperchromatic nuclei, forming cribriform glands. Mitotic figures (blue circles) were frequently identified, with apoptotic bodies and karyorrhectic debris (yellow circles) being readily visible within the epithelium. D, E The MELF pattern was evident at the invasive tumor front, which showed striking stromal desmoplasia and myxoinflammatory responses obscuring the tumor glands. The MELF pattern resulted in an ill-defined lobulating border along the invasive front, with the tumor glands not being apparent at low-power magnification. F A large irregularly dilated gland was partly lined with a single layer of polygonal cells with abundant eosinophilic cytoplasm (lower half and right upper corner). The lining cells were morphologically different from the tumor cells forming the cribriform architecture (upper half). G The conventional UEA component showed a readily identifiable cribriform architecture (upper half), in contrast to the MELF component, which exhibited striking stromal desmoplasia and myxoinflammatory stroma (lower half). H A desmoplastic stromal reaction and lymphoplasmacytic infiltrates surrounded fragmented tumor cells and glands. I The tumor cell clusters in some foci exhibited a squamoid morphology. Randomly scattered individual tumor cells resembled histiocytes (short blue arrows). J, K Lymphovascular invasion was occasionally present. Individual tumor cells (long green arrow), glands (long yellow arrow), and small nests (long red arrow) were observed within the lymphatic vessels. 


\section{Case Reports in Oncology}
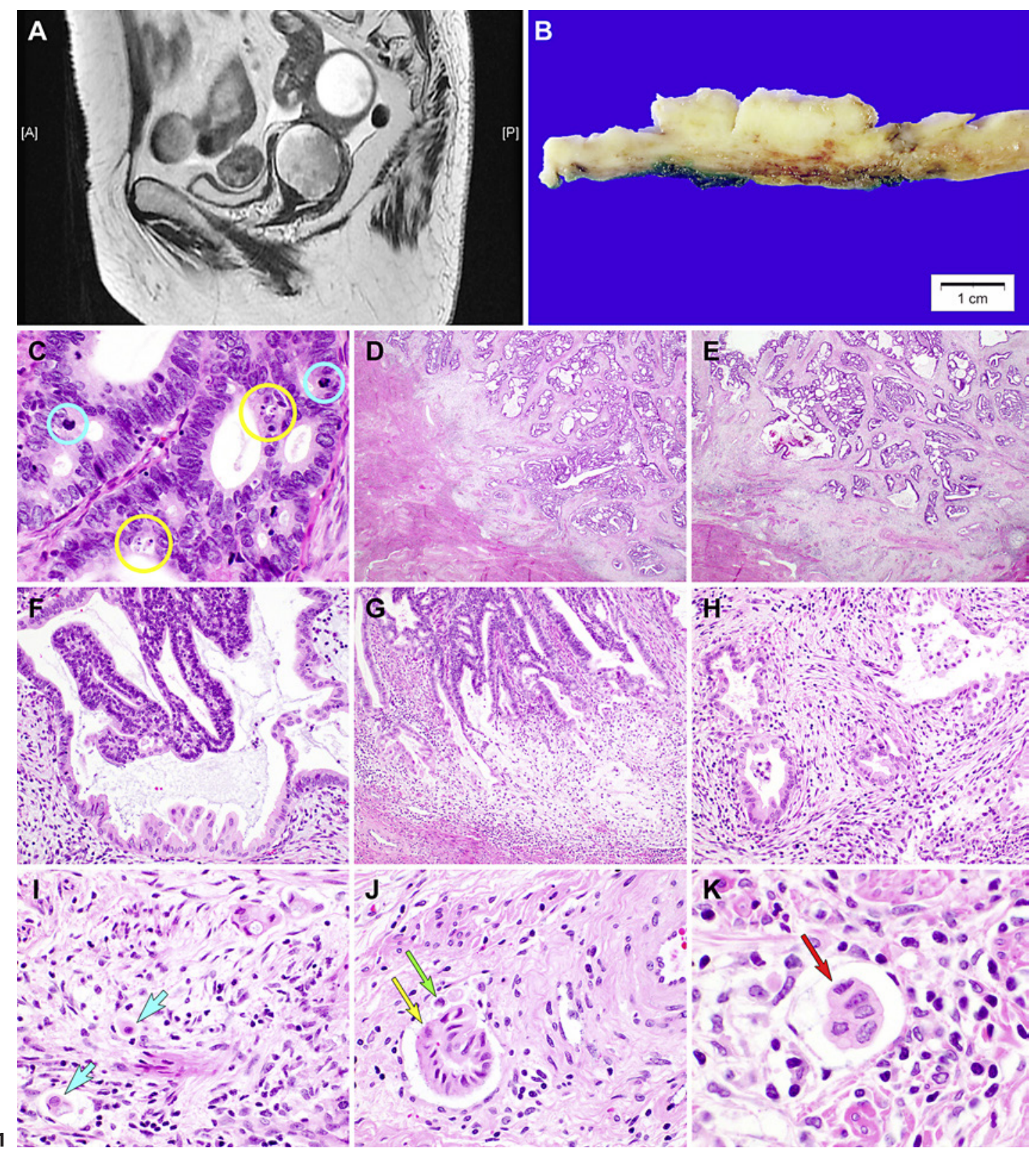

proliferative activity was obvious (Fig. 3E-G). The conventional UEA component showed very high levels of Ki-67 labeling, indicating a high proliferative index, while Ki-67-positive tumor cells in MELF pattern invasion areas were seldomly observed. Tumor cell emboli within lymphatic vessels failed to show any Ki-67 immunoreactivity (Fig. 3H).

For targeted sequencing analysis, total DNA and RNA were sequentially isolated from 10- $\mu \mathrm{m}$ sections of formalin-fixed, paraffin-embedded tissue using RecoverAll Multi-Sample RNA/DNA Isolation Workflow (Thermo Fisher Scientific, Waltham, MA, USA). The DNA and RNA were quantified using a Qubit 2.0 Fluorometer (Thermo Fisher Scientific). DNA and RNA libraries were generated using 10 ng of DNA sample, an Ion S5 Chef System (Thermo Fisher Scientific), and the Oncomine Cancer Research Panel (Thermo Fisher Scientific), which included 161 unique genes, of which 86 oncogenes were analyzed for mutational hotspots 


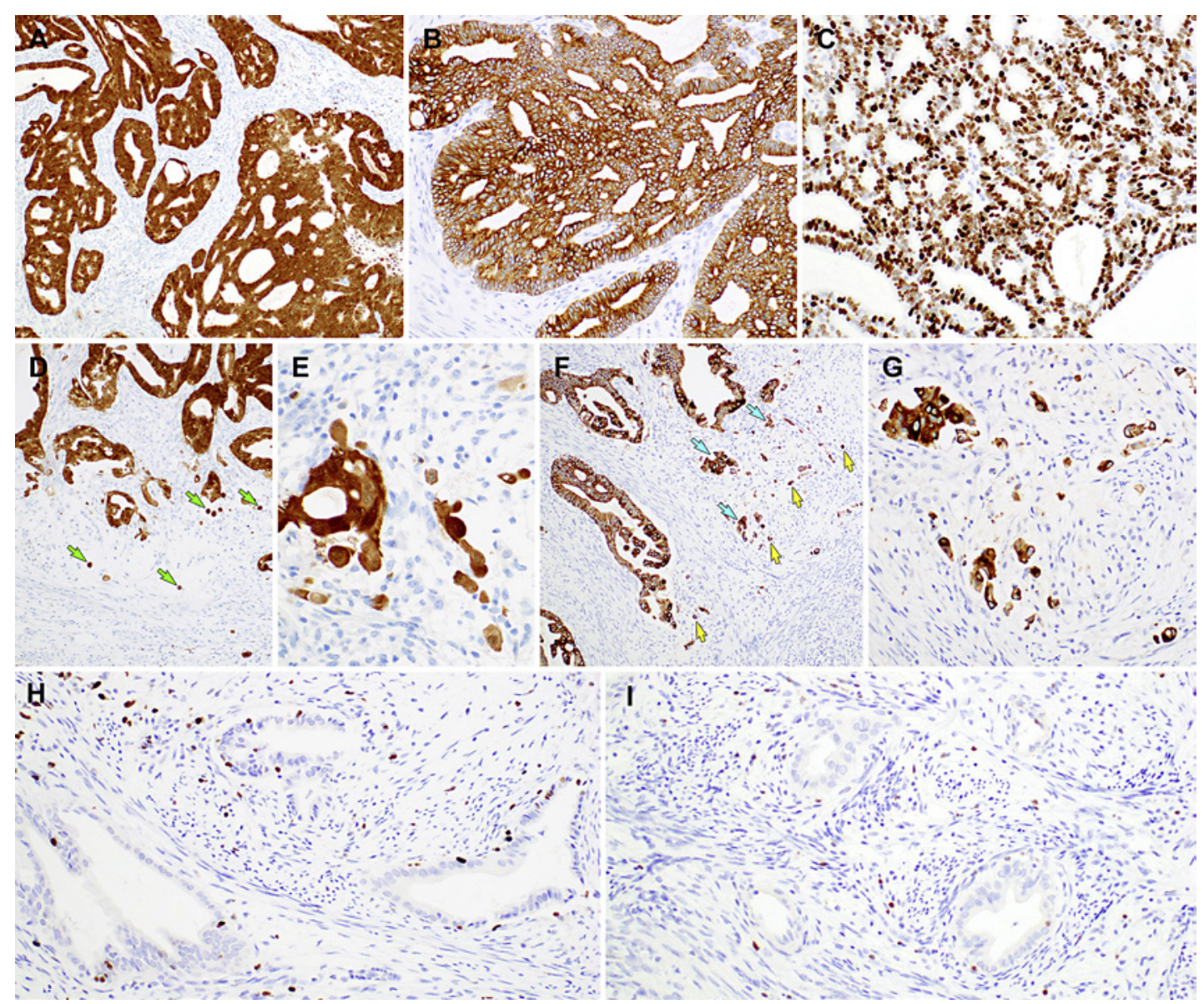

Fig. 2. Immunostaining of usual-type endocervical adenocarcinoma (UEA) showing a microcystic, elongated, and fragmented (MELF) pattern of invasion. A-C UEA displayed block positivity for p16 (A), diffuse and strong CK 7 immunoreactivity (B), and an increased proliferation index (>90\%) as indicated by Ki-67 labeling (C). D, E In the MELF component at the invasive front, p16 immunostaining highlighted randomly scattered individual tumor cells (D, green arrows) and fragmented tumor glands (E). F, G Similar to p16 expression, CK 7 expression was localized to tumor glands (F), irregular-shaped tumor cell clusters (F, blue arrows), and individual tumor cells (G; F, yellow arrows). H, I In contrast to conventional UEA, tumor cells exhibiting a MELF pattern seldomly expressed Ki-67 (proliferative activity <1\%).

and all exons of 48 tumor suppressor genes were evaluated. The Oncomine panel provided the capability to detect copy number variations in 47 genes and fusion drivers in 51 genes. Sequencing was performed using the Ion 540 Kit-Chef (Thermo Fisher Scientific) and Ion S5 Chef System (Thermo Fisher Scientific). Sequencing data consisting of sequencing reads of approximately $200 \mathrm{bp}$ were generated after 500 flow runs. Table 1 summarizes the single nucleotide variations and copy number alterations detected in the MELF pattern and conventional UEA components. UEA exhibiting a MELF pattern harbored pathogenic mutations in ARID1A (c.444C>G), KRAS (c.34G>T), and PIK3CA (c.241G>A, c.1633G>A, and c.2176G>A). The conventional component showed an identical mutational profile to that of the MELF component. Neither copy number alteration nor gene fusion was detected. 


\section{Case Reports in Oncology}

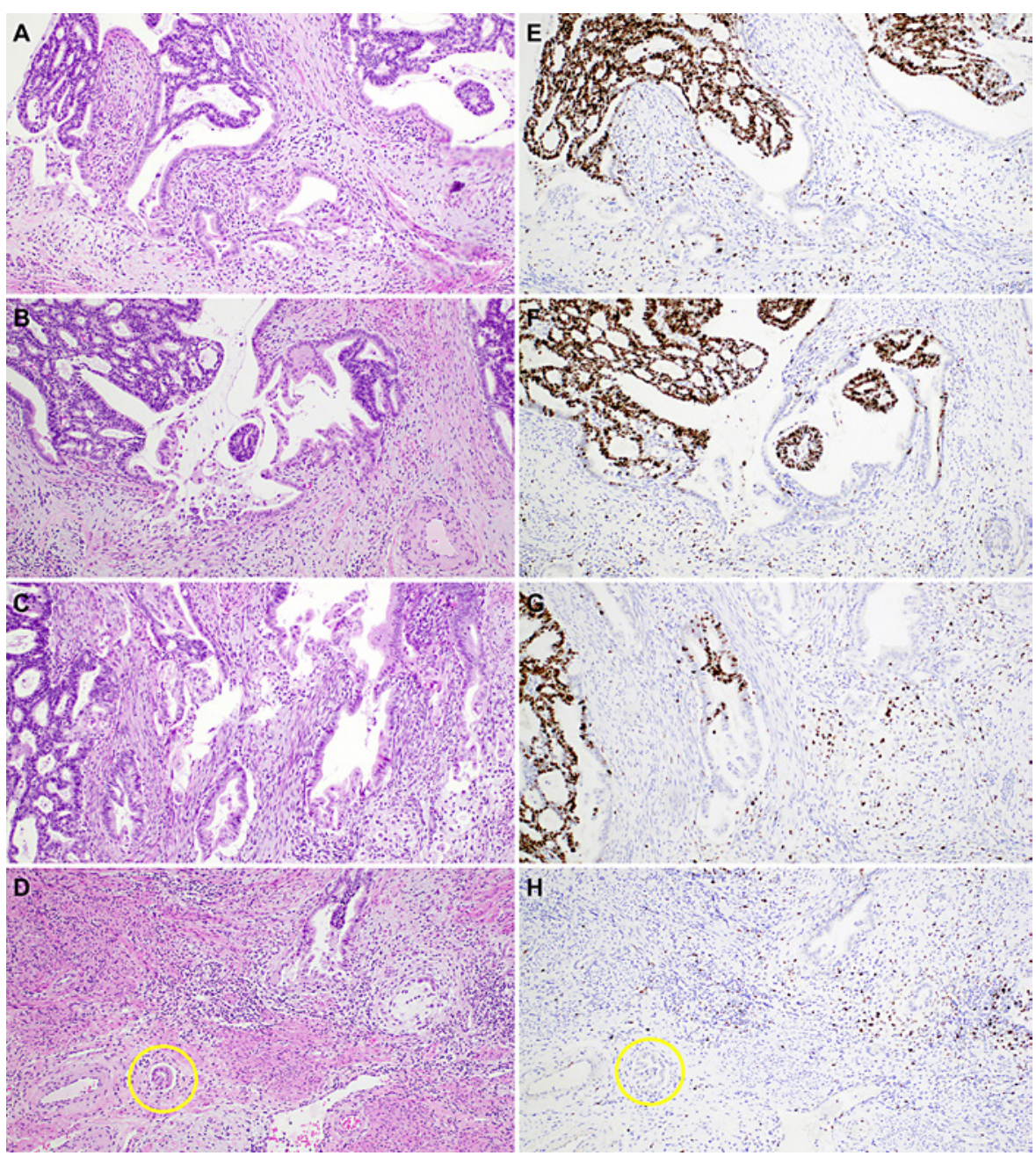

Fig. 3. Ki-67 proliferative activity in transition areas between conventional and microcystic, elongated, and fragmented (MELF) components. A-C Both a cribriform glandular architecture and a MELF pattern were present. D Endolymphatic tumor emboli (yellow circle) were shown at the invasive tumor front. E-G Ki-67 immunostaining highlighted cells in the conventional usual-type endocervical adenocarcinoma component showing noticeably increased proliferative activity. In contrast, few tumor cells were positive for Ki-67 in the MELF component. $\mathbf{H}$ Endolymphatic tumor emboli (yellow circle) were not immunoreactive for Ki-67.

\section{Discussion}

The MELF pattern of stromal invasion represents a highly distinctive morphology of myometrial invasion observed in EECs [4]. It is morphologically characterized by outpouchings of tumor cells from infiltrative tumor glands exhibiting a conventional pattern of invasion. Compared to the conventional invasion pattern, which displays a confluent architecture with cribriform or papillary structures that consist of round-to-oval mucin-depleted glands, the MELF pattern has a more elongated appearance, is lined by a flattened, eosinophilic cytoplasm, and sometimes has a microcystic appearance. These glands fragment into small, irregularly shaped tumor cell clusters and single cells that exhibit a relatively bland eosinophilic or vacuolated histiocyte-like morphology at the deepest tumor invasive front. They are typically associated with a concomitant fibromyxoid stromal reaction and active inflammatory infiltrates, mimicking a microabscess at low-power magnification. In the current study, we 
Table 1. Targeted sequencing results

\begin{tabular}{|c|c|c|c|c|c|c|}
\hline $\begin{array}{l}\text { UEA } \\
\text { component }\end{array}$ & Gene & $\begin{array}{l}\text { Mutation } \\
\text { type }\end{array}$ & $\begin{array}{l}\text { Sequence } \\
\text { change }\end{array}$ & $\begin{array}{l}\text { Predicted } \\
\text { effect }\end{array}$ & $\begin{array}{l}\text { Allele } \\
\text { frequency }\end{array}$ & $\begin{array}{l}\text { Clinical } \\
\text { significance }\end{array}$ \\
\hline \multirow[t]{5}{*}{ MELF } & $A R I D 1 A$ & Nonsense & c. $444 \mathrm{C}>\mathrm{G}$ & p.Tyr148Ter & $27.64 \%$ & Pathogenic \\
\hline & $K R A S$ & Missense & c. $34 \mathrm{G}>\mathrm{T}$ & p.Gly12Cys & $22.01 \%$ & Pathogenic \\
\hline & PIKЗCA & Missense & c. $241 \mathrm{G}>\mathrm{A}$ & p.Glu81Lys & $12.37 \%$ & Pathogenic \\
\hline & PIK3CA & Missense & c. $1633 \mathrm{G}>\mathrm{A}$ & p.Glu545Lys & $17.40 \%$ & Pathogenic \\
\hline & PIKЗCA & Missense & c. $2176 \mathrm{G}>\mathrm{A}$ & p.Glu726Lys & $13.82 \%$ & Pathogenic \\
\hline \multirow[t]{5}{*}{ Conventional } & ARID1A & Nonsense & c. $444 \mathrm{C}>\mathrm{G}$ & p.Tyr148Ter & $71.69 \%$ & Pathogenic \\
\hline & $K R A S$ & Missense & c. $34 \mathrm{G}>\mathrm{T}$ & p.Gly12Cys & $48.22 \%$ & Pathogenic \\
\hline & PIK3CA & Missense & c. $241 \mathrm{G}>\mathrm{A}$ & p.Glu81Lys & $29.84 \%$ & Pathogenic \\
\hline & PIK3CA & Missense & c. $1633 \mathrm{G}>\mathrm{A}$ & p.Glu545Lys & $38.45 \%$ & Pathogenic \\
\hline & PIK3CA & Missense & c. $2176 \mathrm{G}>\mathrm{A}$ & p.Glu726Lys & $37.67 \%$ & Pathogenic \\
\hline
\end{tabular}

UEA, usual-type endocervical adenocarcinoma; MELF, microcystic, elongated, and fragmented.

reported the clinicopathological characteristics of a rare case of UEA with a MELF pattern. Although a recent study has described a MELF pattern in endocervical adenocarcinoma [2], little information is available on its clinicopathological features. For accurate diagnoses, pathologists should be aware that the MELF pattern can also present in UEA.

Previous studies have shown that the MELF pattern is significantly related to more frequent LVI and LNM, as well as worse prognosis for patients with EEC [3, 5, 6]. Zinovkin et al. [5] and Sanci et al. [6] showed that overall survival rates are significantly lower among patients with MELF-positive EEC than among those with MELF-negative EEC. These findings suggest that the MELF pattern may be a potential risk factor for LNM or may serve as a prognostic indicator. However, it remains controversial whether the MELF pattern itself is an independent predictive factor for LNM, due to conflicting data across studies [6-8]. Similarly, the prognostic significance of the MELF pattern has not been completely established, again due to contradictory results. In a study of low-grade EECs [4], the MELF pattern was significantly associated with larger tumor size, myometrial invasion greater than $50 \%$, advanced stage, LVI, and LNM; however, patients whose tumors showed a MELF pattern did not exhibit a worse prognosis than those without a MELF pattern. Other researchers also have not demonstrated significant associations between the MELF pattern and adverse clinical outcomes $[1,9,10]$. With respect to endocervical adenocarcinoma, Segura et al. [2] recently investigated the prognostic value of the MELF pattern in a large cohort of patients. They observed lower overall survival rates and shorter median survival among MELF-positive patients compared to MELFnegative patients, but again, the MELF pattern was not independently associated with worse survival. Although the prognostic implication of the MELF pattern in UEA has not been fully clarified, pathologists should carefully determine the presence of a MELF pattern in UEA, at least because differences in the pathological characteristics of MELF-positive tumors and MELF-negative tumors exist $[2,4]$. Furthermore, additional studies of larger cohorts are necessary to establish the prognostic significance of the MELF pattern in UEA.

It has been documented in EECs that tumor cells at the invasive tumor front exhibiting a MELF pattern are rarely positive for Ki-67 immunostaining, indicating that the MELF pattern may be involved in cellular senescence or growth arrest [4,11]. Kihara et al. [4] suggested that this finding may explain why there are no significant differences in patient survival between MELF-positive and MELF-negative groups, even though LVI and LNM are more frequently observed among patients with MELF-positive EECs. Consistent with these data, we demonstrated in the current study that the MELF component of UEA showed a very low 
proliferation index based on Ki-67 expression compared to the conventional UEA component, which displayed markedly increased Ki-67 expression. The morphological changes may be indicative of progression toward an invasive potential, and it is worth noting that tumor invasion may be associated with reduced cellular proliferation $[12,13]$. Our observation of a significant difference between MELF and conventional UEA components in Ki-67 labeling should be confirmed in further investigations.

To the best of our knowledge, the molecular features of the MELF component have not been explored in previous studies. In the current study, targeted sequencing revealed that the MELF component of UEA harbored multiple pathogenic mutations in ARID1A, KRAS, and PIK3CA, all of which have previously been documented in UEA $[14,15]$. The mutational profiles of the conventional UEA and MELF pattern components were the same. UEA is known to be strongly related to human papillomavirus infection. Consistent with this, we noted that the MELF component also exhibited block p16 positivity, indicating an oncogenic association with high-risk human papillomavirus infection. However, the actual molecular mechanisms associated with the MELF pattern remain unclear.

In summary, we reported a rare case of UEA with a MELF pattern of stromal invasion. The histological features of the MELF pattern observed in the current UEA case were similar to those of EEC. In patients whose tumors display a MELF pattern, careful histological examination, especially regarding LVI and occult LNM, is needed for accurate management. We found a significant difference in Ki-67 expression between the conventional and MELF pattern components, but the mutational profiles were identical. Pathologists should be aware that this unusual invasive pattern may present in UEA to help assure it is distinguished from the conventional invasion pattern.

\section{Statement of Ethics}

Written informed consent for publication was obtained from the patient. This study was reviewed and approved by the Institutional Review Board of Samsung Medical Center (Seoul, Republic of Korea) (2020-07-050).

\section{Conflict of Interest Statement}

The authors have no conflicts of interest to declare.

\section{Funding Sources}

This research was supported by the National Research Foundation of Korea (NRF) grant funded by the Korean government (Ministry of Science and ICT) (2018R1C1B5043725).

\section{Author Contributions}

Sangjoon Choi: conceptualization, data analysis, manuscript drafting, and manuscript editing. Soohyun Hwang: data analysis and manuscript editing. Sung-Im Do: conceptualization, manuscript editing, funding acquisition, and supervision. Hyun-Soo Kim: conceptualization, data collection, data analysis, manuscript drafting, manuscript editing, and supervision. All authors read and approved the final manuscript.

\section{Karger'}




\section{Case Reports in Oncology}

\begin{tabular}{l|l}
\hline Case Rep Oncol 2020;13:1421-1429 \\
\hline DOI: 10.1159/000510441 & $\begin{array}{l}\text { ○ 2020 The Author(s). Published by S. Karger AG, Basel } \\
\text { www.karger.com/cro }\end{array}$ \\
\hline
\end{tabular}

Choi et al.: Endocervical Adenocarcinoma with MELF Invasion Pattern

\section{References}

1 Murray SK, Young RH, Scully RE. Unusual epithelial and stromal changes in myoinvasive endometrioid adenocarcinoma: a study of their frequency, associated diagnostic problems, and prognostic significance. Int J Gynecol Pathol. 2003 Oct;22(4):324-33.

2 Segura SE, Hoang L, Boros M, Terinte C, Pesci A, Aviel-Ronen S, et al. Clinicopathologic association and prognostic value of MELF pattern in invasive endocervical adenocarcinoma (ECA) as classified by IECC. Int J Gynecol Pathol. 2020 Sep;39(5):436-42.

3 Joehlin-Price AS, McHugh KE, Stephens JA, Li Z, Backes FJ, Cohn DE, et al. The microcystic, elongated, and fragmented (MELF) pattern of invasion: a single institution report of 464 consecutive FIGO grade 1 endometrial endometrioid adenocarcinomas. Am J Surg Pathol. 2017 Jan;41(1):49-55.

4 Kihara A, Yoshida H, Watanabe R, Takahashi K, Kato T, Ino Y, et al. Clinicopathologic association and prognostic value of microcystic, elongated, and fragmented (MELF) pattern in endometrial endometrioid carcinoma. Am J Surg Pathol. 2017 Jul;41(7):896-905.

5 Zinovkin DA, Pranjol MZI, Petrenyov DR, Nadyrov EA, Savchenko OG. The potential roles of MELF-pattern, microvessel density, and VEGF expression in survival of patients with endometrioid endometrial carcinoma: a morphometrical and immunohistochemical analysis of 100 cases. J Pathol Transl Med. 2017 Sep;51(5): 456-62.

6 Sanci M, Güngördük K, Gülseren V, Karadeniz T, Kocaer M, Gungorduk O, et al. MELF pattern for predicting lymph node involvement and survival in grade I-II endometrioid-type endometrial cancer. Int J Gynecol Pathol. 2018 Jan;37(1):17-21.

7 Dogan Altunpulluk M, Kir G, Topal CS, Cetiner H, Gocmen A. The association of the microcystic, elongated and fragmented (MELF) invasion pattern in endometrial carcinomas with deep myometrial invasion, lymphovascular space invasion and lymph node metastasis. J Obstet Gynaecol. 2015 May;35(4):397-402.

8 Euscher E, Fox P, Bassett R, Al-Ghawi H, Ali-Fehmi R, Barbuto D, et al. The pattern of myometrial invasion as a predictor of lymph node metastasis or extrauterine disease in low-grade endometrial carcinoma. Am J Surg Pathol. 2013 Nov;37(11):1728-36.

9 Espinosa I, Serrat N, Zannoni GF, Rovira R, D’Angelo E, Prat J. Endometrioid endometrial carcinomas with microcystic, elongated, and fragmented (MELF) type of myoinvasion: role of immunohistochemistry in the detection of occult lymph node metastases and their clinical significance. Hum Pathol. 2017 Dec;70:6-13.

10 Kommoss S, Hartkopf AD, Krämer B, Bunz AK, Grevenkamp F, Kommoss F, et al. Disseminated tumor cells are not associated with established risk factors, L1CAM immunoreactivity and outcome in endometrial carcinoma. J Cancer Res Clin Oncol. 2017 Nov;143(11):2183-8.

11 Stewart CJ, Crook ML, Doherty DA. Micro-anatomical variation in cellular proliferation in endometrial adenocarcinoma, and inverse correlation between Ki67 and cytokeratin 7 expression. Histopathology. 2010 Jul; 57(1):46-54.

12 Jung A, Schrauder M, Oswald U, Knoll C, Sellberg P, Palmqvist R, et al. The invasion front of human colorectal adenocarcinomas shows co-localization of nuclear beta-catenin, cyclin D1, and p16INK4A and is a region of low proliferation. Am J Pathol. 2001 Nov;159(5):1613-7.

13 Nilsson H, Ely D, Friberg P, Karlström G, Folkow B. Effects of high and low sodium diets on the resistance vessels and their adrenergic vasoconstrictor fibre control in normotensive (WKY) and hypertensive (SHR) rats. Acta Physiol Scand. 1985 0ct;125(2):323-34.

14 Chung TK, Van Hummelen P, Chan PK, Cheung TH, Yim SF, Yu MY, et al. Genomic aberrations in cervical adenocarcinomas in Hong Kong Chinese women. Int J Cancer. 2015 Aug;137(4):776-83.

15 Hodgson A, Amemiya Y, Seth A, Cesari M, Djordjevic B, Parra-Herran C. Genomic abnormalities in invasive endocervical adenocarcinoma correlate with pattern of invasion: biologic and clinical implications. Mod Pathol. 2017 Nov;30(11):1633-41. 